\title{
Non-Existence of Recursive Equilibria on Compact State Spaces When Markets are Incomplete
}

\author{
First Draft: September, 2000 \\ This Version: April, 2002 \\ Tom Krebs * \\ Brown University ${ }^{\dagger}$
}

\begin{abstract}
This paper analyzes one-good exchange economies with two infinitely-lived agents and incomplete markets. It is shown that there are no recursive (Markov) equilibria for which borrowing (debt) constraints never bind if the state space of exogenous and endogenous variables is a compact subset of $\mathbb{R}^{n}$. Moreover, for large enough (but finite) borrowing limits, no recursive equilibrium with compact state space exists. These non-existence results hold for any economy satisfying the following standard assumptions: preferences are additively separable across time and states; the one-period utility function is time- and state-independent and unbounded from below; endowments are bounded and follow a Markov chain (finite support) with stationary transition matrix; there is some idiosyncratic risk and no aggregate risk. The same non-existence results hold for any number of agents if in addition the marginal utility functions of all agents are convex. Finally, non-existence holds for any number of agents and any Markov endowment chain (with or without aggregate risk) if all agents have identical utility functions of the CRRA-type (homothetic preferences) with degree of relative risk aversion greater than or equal to one.
\end{abstract}

JEL classification codes: D50,D52

Keywords: Recursive (Markov) Equilibrium, Incomplete Markets, (Non)-Existence

*This paper previously circulated under the title "On the Non-Existence of Recursive (Markov) Equilibria When Markets Are Incomplete". I would like to thank Herakles Polemarchakis for extensive conversations on the topic and Paolo Siconolfi for several suggestions that clarified and generalized my previous results substantially. I also wish to thank for helpful comments David Cass, Darrell Duffie, Tomo Nakajima, Martine Quinzii, Rajiv Vohra, Bill Zame, an anonomous referee and an associate editor of this Journal, and seminar participants at Brown University, the University of Pennsylvania, the Midwest Economic Theory Conference, Fall 2000, the Economic Theory Conference in Ischia, 2001, and the SITE-GE workshop, 2001. All errors are mine.

${ }^{\dagger}$ Department of Economics, Box B, Providence, RI 02912. e-mail: tom_krebs@brown.edu. web: www.econ.brown.edu/ ${ }^{\sim}$ tkrebs 


\section{Introduction}

A large part of macroeconomics deals with recursive equilibria (Markov equilibria) in recursive economies (Cooley and Prescott, 1995, Stokey and Lucas, 1989, Ljungqvist and Sargent, 2000). For exchange economies with one infinitely-lived agent, Lucas (1978) has shown the existence of recursive equilibria (RE) with a state space that only contains exogenous variables. For exchange economies with a finite number of infinitely-lived agents and incomplete markets, Duffie, Geanakoplos, Mas-Collel, and McLennan (1994) have shown the existence of RE with a compact state space that includes the exogenous variables and the endogenous variables consumption, asset prices, and portfolio holdings. In order to prevent agents from entering into Ponzi schemes, Duffie et.al. (1994) introduce an explicit borrowing (short-sale) constraint that may bind in equilibrium. ${ }^{1}$ For the same class of economies, Hernandez and Santos (1996), Levine and Zame (1996), and Magill and Quinzii $(1994,1996)$ have shown the (generic) existence of sequential equilibria (not necessarily recursive) with explicit borrowing constraints that never bind in equilibrium. This paper asks whether the same is true for RE. That is, can we find RE (the focus of Duffie et.al., 1994) with explicit borrowing constraints that never bind (the focus of Hernandez and Santos,1996, Levine and Zame,1996, and Magill and Quinzii,1994,1996)? ${ }^{2}$

A RE is defined by a state space, a law of motion for the endogenous state variable(s),

\footnotetext{
${ }^{1}$ This terminology follows Magill and Quinzii (1994) and most of the macroeconomic literature. Duffie et.al. (1994) only consider assets in positive net-supply and rule out short-selling (borrowing) by assumption. For this type of borrowing constraint, they prove the existence of a stationary (ergodic) Markov equilibrium (ME), which implies the existence of a stationary (ergodic) RE. See Duffie et.al. (1994) and this paper for a discussion of the relationship between RE and ME. Becker and Zilcha (1997) prove the existence of a stationary equilibrium for production economies with only aggregate productivity shocks (no idiosyncratic risk), no borrowing, and an endogenous state space that contains only the wealth distribution.

${ }^{2}$ The introduction of ad-hoc borrowing (short-sale) constraints by Duffie et.al. (1994) is reminiscent of Radner's (1972) lower bound on short-sales to ensure existence of equilibrium in finite-horizon economies, which was subsequently criticized by Hart (1975). Notice, however, that in contrast to Hart (1975) the non-existence result discussed in this paper is not caused by an asset demand (demand for borrowing) that goes to infinity when the borrowing constraint is gradually relaxed.
} 
and a (vector-valued) function mapping current states into current outcomes (Cooley and Prescott, 1995). Similarly, a Markov equilibrium (ME) is defined by a state space (selfjustified set) and an expectations correspondence (Duffie et.al., 1994). Thus, the state space is one of the basic ingredients of a RE (ME). This paper shows that for a large class of exchange economies with incomplete markets, the state space cannot be a compact subset of $\mathbb{R}^{n}$ if borrowing constraints never bind (and all functions are continuous). In other words, no RE (ME) with compact state space and non-binding borrowing constraints exists. In particular, no RE (ME) with finite state space and non-binding borrowing constraints exists even if the Markov process of exogenous variables has finite support. From the non-existence of RE (ME) with compact state space and non-binding borrowing constraints it follows that for large enough (but finite) borrowing limits, no RE (ME) with compact state space exists. Put differently, as we gradually relax the explicit borrowing constraints used by Duffie et.al. (1994) to prevent Ponzi schemes, we move from existence to non-existence.

Both Duffie et.al. (1994) and the computational literature on RE with incomplete markets (Aiyagari, 1994, Huggett, 1993, Lucas and Heaton, 1996, and Krusell and Smith, 1998) confine attention to RE (ME) with explicit borrowing constraints and compact state space. ${ }^{3}$ This paper shows that under the maintained assumption of a compact state space, the explicit borrowing constraint used by Duffie et.al. (1994) and the computational literature is necessarily ad-hoc in the sense that it always prevents a solvent agent from borrowing an additional dollar at some date-event. In other words, the borrowing constraint always introduces a market imperfection in addition to market incompleteness, and this literature has therefore relied on a framework that does not allow for a clear separation of market incompleteness from other market imperfections. Furthermore, any future work on the

\footnotetext{
${ }^{3}$ The compactness of the state space is essential for many of the arguments put forward in Duffie et.al. (1994). In particular, the existence of a RE (ME) with compact state space implies, under an additional convexity assumption on the expectations correspondence, that a stationary (ergodic) RE (ME) exists. The convexity assumption is satisfied for the class of economies analyzed in this paper.
} 
existence of RE (ME) with non-binding borrowing constraints must either abandon the compactness assumption (and therefore go beyond the methods discussed by Duffie et.al., 1994) or change the definition of RE. ${ }^{4}$

The basic non-existence argument for an economy without aggregate risk runs as follows. First, for utility functions that are unbounded from below, zero consumption can never be an equilibrium outcome. ${ }^{5}$ Second, for equilibria with strictly positive consumption and non-binding borrowing constraints, market clearing and agents' first-order conditions (Euler equations) taken together imply that if one agent's equilibrium consumption attains its minimum (maximum), then consumption of this agent must be equal to this minimial (maximal) value for all future dates (and future shock realizations). Third, no agent's equilibrium consumption can be constant if markets are incomplete. In other words, the temporary equilibrium conditions require that future consumption is constant once minimal (maximal) consumption is achieved, but the condition of overall equilibrium (the present value budget constraint) requires that consumption always exhibits some fluctuations. Thus, minimal consumption can never be achieved. If the consumption allocation is a part of the (endogenous) state space, then this immediately implies the non-existence of RE with compact state space. If the consumption allocation is not a part of the state space, then an additional continuity assumption implies non-existence. ${ }^{6}$

\footnotetext{
${ }^{4}$ For example, Duffie et.al. (1994) require that the equilibrium conditions hold for all possible current states (for all elements of the state space), which could be changed to the requirement that the equilibrium conditions hold for almost all current states.

${ }^{5}$ One might conjecture that an Inada condition is sufficient to rule out zero consumption, but this conjecture is not correct if an agent's initial debt is so large that his initial choice set is a singleton. See footnote 7 for a more detailed discussion of this issue. In the case of CRRA-preferences, the unboundedness assumption means that the degree of relative risk aversion is greater than or equal to one. Risk aversion coefficients between one and four are the focus of the computational literature (Aiyagari, 1994, Huggett, 1993, Lucas and Heaton, 1996, and Krusell and Smith, 1998).

${ }^{6}$ The argument has the flavor of the reasoning employed by Atkeson and Lucas (1992) and Thomas and Worrall (1990) to show that the (constrained)-efficient cross-sectional distribution of consumption diverges in economies with private information. Their analysis, however, confines attention to economies with a
} 
The non-existence result shown in this paper holds for any two-agent incomplete-markets economy satisfying the following standard assumptions: preferences are additively-separable across time and states and one-period utility functions are time- and state-independent; the endowments are bounded and follow a Markov chain (finite support) with stationary transition matrix; there is some idiosyncratic risk and no aggregate risk. Moreover, this paper follows Duffie et.al. (1994) and assumes that the one-period utility function is unbounded from below. If in addition the marginal utility functions of all agents are convex, then the non-existence result holds for any number of agents. Finally, non-existence holds for any number of agents and any Markov chain of endowments (with or without aggregate risk) if agents have identical homothetic preferences (CRRA utility functions) with degree of relative risk aversion greater than or equal to one, the case most frequently encountered in macroeconomics.

Clearly, the class of economies analyzed in this paper is non-pathological. Hence, it is not the structure of the economy, but the structure of the equilibrium concept in conjunction with the compactness assumption that is driving the non-existence result. Indeed, for the same class of economies, Duffie et.al. (1994) prove the existence of RE (ME) with ad-hoc borrowing constraints (no borrowing) and compact state space. Further, if there is only one agent or markets are complete, there are RE with finite (and therefore compact) state space and non-binding borrowing constraints (Lucas, 1978, and this paper), namely the equilibria corresponding to the Pareto efficient consumption allocations.

There are several papers dealing with the issue of non-existence of RE (Markov equilibria) in general equilibrium models. For OLG economies with multiple goods, Spear (1985) has shown that no RE (Markov equilibrium) with finite state space exists. For economies with infinitely-lived agents and incomplete markets, Kehoe and Levine (2001) and Kuebler

continuum of agents and constant interest rate, whereas this paper's focus is on economies with finitelymany agents and possibly time-varying interest rate (asset price). 
and Schmedders (2001) show (generic) non-existence of RE when the state space only contains the exogenous state (finite state space). Kuebler and Schmedders (2002) provide a numerical example (one particular economy) of non-existence of RE with non-binding borrowing constraints (but not necessarily compact state space) when the endogenous part of the state space includes the wealth distribution, but in contrast to this paper they argue that non-existence is related to multiplicity of equilibrium. Finally, Santos (2000) discusses the non-existence of RE in a deterministic economy with externalities.

\section{The Model}

\section{a) The Economy}

We consider a discrete-time, infinite-horizon exchange economy. The economy is populated by two (types of) infinitely-lived agents. Time is indexed by $t=0,1,2, \ldots$ and agents by $i=1,2$.

There is an exogenous Markov process, $\left\{s_{t}\right\}$, with state space, $\mathbf{S}$, and stationary transition function $\pi$. We denote a typical element of $\mathbf{S}$ by $s_{t}$ or simply $s$. A (partial) history of exogenous states up to time $t$ is denoted by $s^{t}=\left(s_{0}, s_{1}, \ldots, s_{t}\right) \in \mathbf{S}^{\mathbf{t}+\mathbf{1}}$ and the probability that $s_{t+1} \in \mathbf{S}$ (respectively $s^{\prime} \in \mathbf{S}$ ) occurs in period $t+1$ if $s_{t} \in \mathbf{S}$ (respectively $s \in \mathbf{S}$ ) has occured in period $t$ is denoted by $\pi\left(s^{\prime} \mid s\right)$ (respectively $\pi\left(s_{t+1} \mid s_{t}\right)$ ). Finally, we assume that in period 0 one state, $s_{0}$, occurs with probability one. In the formal analysis we assume that $\mathbf{S}$ is finite, $\mathbf{S} \equiv\{1, \ldots, S\}$. That is, $\left\{s_{t}\right\}$ is a Markov chain. Each Markov chain, $\left\{s_{t}\right\}$, with initial state $s_{0}$, state space $\mathbf{S}$, and transition matrix $\pi$ induces an event tree with nodes (date-events) $s^{t}$ in the canonical way. We denote the set of successors of $s^{t}$ by $\mathbf{D}\left(s^{t}\right)$.

We assume that the exogenous state space is the Cartesian product of two sets, $\mathbf{S} \equiv \mathbf{\Sigma} \times \mathbf{Z}$, so that $s=(\sigma, z)$. Below we introduce the assumption that asset payoffs, one-period utility 
functions, and endowments are independent of $\sigma$. Thus, we can interpret $z$ as a fundamental shock and $\sigma$ as a signal (news). If $\pi\left(z^{\prime} \mid \sigma, z\right)$ depends non-trivially on $\sigma$, the signal is useful in predicting future fundamentals. Otherwise, the signal is a sunspot variable. The introduction of an additional signal variable, $z$, generalizes the non-existence result and also helps relate the present framework to the framework used by Duffie et.al.,1994 (see below).

There is one perishable consumption good. Agent $i^{\prime} s$ initial endowment of the consumption good (labor income) is defined by a (time-invariant) function $e_{i}: \mathbf{Z} \rightarrow \mathbb{R}_{+}$whose values are denoted by $e_{i}(z)$. The function $e_{i}$ in conjunction with the Markov shock process induces a Markov endowment process in the canonical way.

A consumption plan of agent $i$ is a sequence of functions, $\left\{c_{i t}\right\}$, with $c_{i t}: \mathbf{S}^{\mathbf{t}+\mathbf{1}} \rightarrow \mathbb{R}_{+}$. The value, $c_{i t}\left(s^{t}\right)$, of the function $c_{i t}$ is simply the consumption level of agent $i$ at node $s^{t}=\left(s_{0}, s_{1}, \ldots, s_{t}\right)$. We restrict consumption choices to the space of all sequences that are bounded in the sup-norm: $\left\|\left\{c_{i t}\right\}\right\|=\sup _{t, s^{t}} c_{i t}\left(s^{t}\right)<\infty$. Clearly, a consumption plan can also be defined as one function mapping nodes, $s^{t}$, into consumption levels, $c_{t}\left(s^{t}\right)$. Since the set of all nodes is countable, this means that the consumption space can be identified with $l_{+}^{\infty}$.

Agent $i^{\prime} s$ preferences over consumption plans allow for a time-additive expected utility representation

$$
U_{i}\left(\left\{c_{i t}\right\}\right)=\sum_{t=0}^{\infty} \beta^{t} \sum_{s^{t}} \pi\left(s^{t}\right) u_{i}\left(c_{i t}\left(s^{t}\right)\right),
$$

where $\pi\left(s^{t}\right)=\pi\left(s_{t} \mid s_{t-1}\right) \pi\left(s_{t-1} \mid s_{t-2}\right) \ldots \pi\left(s_{1} \mid s_{0}\right)$ (recall that only one state, $s_{0}$, occurs with positive probability in period 0 ). In (1) we assumed that the pure discount factor, $\beta$, is the same for both agents. Although this assumption is not essential for our non-existence result, it shortens the proofs and provides for a simple benchmark (constant consumption) in the complete-market case with no aggregate risk. 
There is one long-lived asset in positive net-supply. The asset is real in the sense that it promises the delivery of (state-contingent) quantities of the good. The asset payoff structure is described by a time-invariant function, $d: \mathbf{Z} \rightarrow \mathbb{R}_{+}$, with values denoted by $d(z)$. At time $t=0$, agent $i$ has an initial asset endowment of $\theta_{i 0} \in \mathbb{R}$, which is not a choice variable. We normalize the aggregate supply of the asset to one: $\theta_{10}+\theta_{20}=1$.

In short, an economy $\mathcal{E}=<\mathbf{S}, \pi, \beta, u, e, \theta_{0}, d>$ consists of a Markov process with finite state space $\mathbf{S}$ and stationary transition matrix $\pi$, preferences defined by a common discount factor $\beta$ and one-period utility functions $u=u_{1}, \ldots, u_{I}$, endowments defined by functions $e=e_{1}, \ldots, e_{I}$ and initial values $\theta_{0}=\theta_{10}, \ldots, \theta_{I 0}$, and asset payoffs defined by functions $d=d_{1}, \ldots, d_{I}$.

We make the following assumptions on endowments and preferences:

Assumption 1. All transitions have strictly positive probability: $\pi\left(s^{\prime} \mid s\right)>0 \forall s, s^{\prime}$. There is some risk, that is, there exist shocks $z, z^{\prime}$ with $e_{i}(z) \neq e_{i}\left(z^{\prime}\right)$. Asset payoffs are always strictly positive: $\forall z: d(z)>0$.

Assumption 2. Each agent's one-period utility function, $u_{i}: \mathbb{R}_{++} \rightarrow \mathbb{R}$, is twice continuously differentiable, strictly increasing, strictly concave, and unbounded from below.

Remark 1. The assumption $\pi\left(s^{\prime} \mid s\right)>0$ ensures that all nodes have a positive probability of occurrence. Thus, we do not have to distinguish between statements that hold for all nodes and statements that hold almost surely. Notice that we do not impose a positivity assumption on net-endowments, $\theta_{i 0}+e_{i}(z)$, because our non-existence result does not require it. The unboundedness assumption on utility is also made by Duffie et.al. (1994) and ensures that zero consumption is never an equilibrium choice. See footnote 7 for a discussion why the alternative assumption of an Inada condition might not suffice for the non-existence argument. CRRA-preferences with a coefficient of relative risk aversion greater or equal to 
one have the property that the utility function is unbounded from below.

The basic argument for non-existence makes the following assumption:

Assumption 3. There is no aggregate risk: $d(z)+e_{1}(z)+e_{2}(z)=\omega$.

Finally, we state an assumption which rules out that the trading of one asset is sufficient to implement the (Pareto efficient) constant allocation when there are two agents. Clearly, this assumption is generically satisfied.

Assumption 4. Markets are effectively incomplete: there are no numbers $c_{1}, \theta_{1}$ with $0<c_{1}<\omega$ so that $c_{1}-e_{1}(z)=d(z) \theta_{1}$ for all $z$ and no numbers $c_{2}, \theta_{2}$ with $0<c_{2}<\omega$ so that $c_{2}-e_{2}(z)=d(z) \theta_{2}$ for all $z$.

\section{b) Equilibrium}

Agents have the opportunity to trade the asset in a sequence of competitive markets. Let $q_{t}$ stand for the price of one unit of the asset in period $t$ and $\theta_{i t}$ for agent $i^{\prime} s$ beginningof-period holding (before asset trading) of the asset in period $t$. The asset price system is defined by a sequence of functions, $\left\{q_{t}\right\}$, with $q_{t}: \mathbf{S}^{\mathbf{t}+\mathbf{1}} \rightarrow \mathbb{R}_{+}$. A portfolio (trading) plan of agent $i$ is a sequence of functions, $\left\{\theta_{i t}\right\}$, with $\theta_{i, t+1}: \mathbf{S}^{\mathbf{t}+\mathbf{1}} \rightarrow \mathbb{R}$. To rule out Ponzi-schemes, we introduce an explicit debt (borrowing) constraint, $M \geq 0$. Thus, a budget-feasible consumption and portfolio plan has to satisfy the following sequential budget constraint augmented by an explicit borrowing constraint:

$$
\begin{aligned}
\forall t, s^{t}: c_{i t}\left(s^{t}\right)-e_{i}\left(z_{t}\right) & =\left[d\left(z_{t}\right)+q\left(s^{t}\right)\right] \theta_{i t}\left(s^{t-1}\right)-q_{t}\left(s^{t}\right) \theta_{i, t+1}\left(s^{t}\right) \\
q_{t}\left(s^{t}\right) \theta_{i, t+1}\left(s^{t}\right) & \geq-M .
\end{aligned}
$$

We adopt the convention $\theta_{i 0}\left(s^{-1}\right)=\theta_{i 0}$ so that equation (2) also holds for $t=0$.

The are several ways of ruling out Ponzi-schemes, and imposing an explicit bound on 
debt, $M$, is one alternative. However, for large enough $M$ the set of equilibria implied by (2) is the same as the set of equilibria that would result if one of the alternatives discussed in Hernandez and Santos (1996), Levine and Zame (1996), and Magill and Quinzii $(1994,1996)$ was chosen. For example, Hernandez and Santos (1996) show that six different ways of writing down the budget constraint lead to the same budget set (and therefore the same set of equilibria) if asset prices and payoffs are arbitrage-free and if the expected present value of life-time endowment of every agent is finite under some state price process.

Market clearing reads:

$$
\forall t, s^{t}: \theta_{1, t+1}\left(s^{t}\right)+\theta_{2, t+1}\left(s^{t}\right)=1, c_{1 t}\left(s^{t}\right)+c_{2 t}\left(s^{t}\right)=e_{1}\left(z_{t}\right)+e_{2}\left(z_{t}\right)+d\left(z_{t}\right) .
$$

Introduce the functions $\theta_{t+1}: \mathbf{S}^{\mathbf{t}+\mathbf{1}} \rightarrow \mathbb{R}^{2}$ with $\theta_{t+1}\left(s^{t}\right)=\left(\theta_{1, t+1}\left(s^{t}\right), \theta_{2, t+1}\left(s^{t}\right)\right)$ and $c_{t}: \mathbf{S}^{\mathbf{t}+\mathbf{1}} \rightarrow \mathbb{R}_{+}^{2}$ with $c_{t}\left(s^{t}\right)=\left(c_{1 t}\left(s^{t}\right), c_{2 t}\left(s^{t}\right)\right)$.

The following definition of a sequential equilibrium (SE) is standard:

Definition 1. For an economy $\mathcal{E}=<\mathbf{S}, \pi, \beta, u, e, \theta_{0}, d>$, a sequential equilibrium (SE) is a list of sequences, $\left\{q_{t}\right\},\left\{c_{t}\right\},\left\{\theta_{t}\right\}$, so that:

i) For given asset prices $\left\{q_{t}\right\}$, the consumption and trading plan, $\left\{c_{i t}\right\},\left\{\theta_{i t}\right\}$, of each agent $i$ maximizes expected life-time utility (1) subject to the sequential budget constraint (2).

ii) Market clearing (3) holds.

With the assumptions made so far (concave utility and convex choice set), a standard argument shows that Euler equations and transversality condition together are sufficient conditions for individual utility maximization. Although we allowed for the possibility of unbounded asset prices and portfolio choices, below we show that in equilibrium they will be bounded, which implies that any transversality condition is automatically satisfied. Hence, in equilibrium the Euler equation is a sufficient condition for individual utility maximization. Further, in equilibrium the Euler equation holds with equality when borrowing constraints 
are not binding because the unboundedness of utility from below rules out zero consumption as an equilibrium choice. ${ }^{7}$ To summarize, a SE with non-binding borrowing constraints is a list of bounded sequences, $\left\{q_{t}\right\},\left\{c_{t}\right\},\left\{\theta_{t}\right\}$, solving (goods market clearing is implied by asset market clearing and the budget constraint)

$$
\begin{aligned}
\forall i, \forall t, s^{t}: c_{i t}\left(s^{t}\right)-e_{i}\left(z_{t}\right) & =\left[d\left(z_{t}\right)+q_{t}\left(s^{t}\right)\right] \theta_{i t}\left(s^{t-1}\right)-q_{t}\left(s^{t}\right) \theta_{i, t+1}\left(s^{t}\right) \\
\forall i, \forall t, s^{t}: \quad q_{t}\left(s^{t}\right) u_{i}^{\prime}\left(c_{i t}\left(s^{t}\right)\right) & =\beta E\left[\left(d\left(z_{t+1}\right)+q_{t}\left(s^{t+1}\right)\right) u_{i}^{\prime}\left(c_{i, t+1}\left(s^{t+1}\right)\right) \mid s^{t}\right] \\
\forall t, s^{t}: \quad \theta_{1, t+1}\left(s^{t}\right)+\theta_{2, t+1}\left(s^{t}\right) & =1 .
\end{aligned}
$$

We now turn to a discussion of recursive equilibria (RE). Introduce an endogenous state space $\mathbf{X}$ with elements $x$ and time-invariant functions, $f: \mathbf{X} \times \mathbf{S}^{2} \rightarrow \mathbf{X}, q: \mathbf{X} \times \mathbf{S} \rightarrow \mathbb{R}_{+}$, $c: \mathbf{X} \times \mathbf{S} \rightarrow \mathbb{R}_{+}^{2}$, and $\theta: \mathbf{X} \times \mathbf{S} \rightarrow \mathbb{R}^{2}$. For simplicity, we assume that $\theta_{t}$ is always one component of $x_{t}$. Since this paper shows the non-existence of RE, this assumption is without loss of generality. The functions $f, q, c, \theta$ in conjunction with an initial condition $\left(x_{0}, s_{0}\right)$ generate (define) sequences $\left\{x_{t}\right\},\left\{q_{t}\right\},\left\{c_{t}\right\}$, and $\left\{\theta_{t}\right\}$ through the recursive formula

$$
\begin{aligned}
x_{t+1}\left(s^{t+1}\right) & =f\left(x_{t}\left(s^{t}\right), s_{t}, s_{t+1}\right) \\
q_{t}\left(s^{t}\right) & =q\left(x_{t}\left(s^{t}\right), s_{t}\right) \\
c_{t}\left(s^{t}\right) & =c\left(x_{t}\left(s^{t}\right), s_{t}\right) \\
\theta_{t+1}\left(s^{t}\right) & =\theta\left(x_{t}\left(s^{t}\right), s_{t}\right) .
\end{aligned}
$$

The function $f$ describes the law of motion for the endogenous state variable(s).

To simplify notation, we will assume that $x_{t}$ only contains "current "endogenous variables, that is, we assume that the largest possible endogenous state is $x_{t}$ is $x_{t}=\left(q_{t}, c_{t}, \theta_{t}\right)$. However,

\footnotetext{
${ }^{7}$ Since we do not require net-endowments to be strictly positive, zero consumption could arise in equilibrium even if the utility function satisfies an Inada condition (but is bounded from below): if an agent begins his life with so much debt that his net-endowment is zero, $\theta_{i 0}+e_{i}\left(z_{0}\right)=0$, then his choice set at the initial node is a singleton and no Euler equation has to hold for this agent at the initial node (any further increase in debt will violate the budget constraint and the agent is forced to consume $c_{i 0}=0$ ).
} 
the non-existence results proved in this paper immediately extend to the case in which $x_{t}$ contains a finite number of current and past endogenous variables and a finite number of past shocks.

When the point of departure is the notion of SE, it seems tempting to define a recursive equilibrium (RE) as a SE, $\left\{q_{t}\right\},\left\{c_{t}\right\},\left\{\theta_{t}\right\}$, generated by the recursive formula (5) and an initial condition $\left(x_{0}, s_{0}\right)$. However, in this paper we define a RE as a family of SE, namely the family of SE generated by the recursive formula (5) when any $(x, s) \in \mathbf{X} \times \mathbf{S}$ is a possible initial state. We therefore introduce the following concept of a RE:

Definition 2. For an economy $\mathcal{E}=<\mathbf{S}, \pi, \beta, u, e, \theta_{0}, d>$, a recursive equilibrium (RE) is a family of SE generated by a set of initial conditions, $\mathbf{X} \times \mathbf{S}$, and time-invariant functions $f, q, c, \theta$ with domain $\mathbf{X} \times \mathbf{S}$.

Most work of the work on RE (see, for example, Prescott and Cooley, 1995) immediately formulates the individual optimization problem in terms of the corresponding Bellman equation, which automatically implies that a RE corresponds to a family of SE (in accordance with the above definition). Put differently, the solution to the Bellman equation corresponds to a family of solutions to the corresponding optimization problem, one (in our case of strictly concave utility function and convex choice sets) for each initial condition. In addition to this "mathematical reason", there are at least two "non-mathematical"reasons for not identifying a RE with one SE for a fixed initial state. First, such a definition would imply that a RE depends on the initial condition, and therefore exhibits some sort of infinite memory. Second, the set of possible future realizations of the state may explicitly depend on time, again a feature which seems to run counter to the notion of recursivity.

Each RE defines a (time-homogeneous) joint Markov process over endogenous and exogenous variables. Such a Markov process satisfying all equilibrium conditions is called a 
Markov equilibrium (Duffie et.al., 1994). Duffie et.al. (1994) do not define a Markov equilibrium indirectly through a $\mathrm{RE}$, but directly through a state space and an expectations correspondence. If one allows for signal variables, $\sigma_{t}$, then any Markov equilibrium can be represented as a RE (Duffie et.al., 1994).

The previous literature has studied the following special cases of the general framework. In the case of one representative agent (Lucas, 1978) or complete markets (proposition 1 below), there are RE for which equilibrium consumption and asset prices only depend on the exogenous state $s$, and for which the portfolio holdings of the long-lived asset are constant. Thus, we can choose as state space the exogenous set $\mathbf{S}$, which is finite and therefore compact. The quantitative literature on incomplete markets (Aiyagari, 1994, Heaton and Lucas, 1996, Huggett, 1993, Krusell and Smith, 1998) in general uses a minimal endogenous state space, that is, the only endogenous state variable is the wealth distribution: $x_{t}=\theta_{t}$ or $x_{t}=q_{t-1} \theta_{t}$. Moreover, the possibility of additional signal variables is often neglected $\left(s_{t}=z_{t}\right)$. Finally, Duffie et.al. (1994) use $x_{t}=\left(\theta_{t}, c_{t}, q_{t}\right)$ as the endogenous state of the economy (they also include $\theta_{t+1}$ in the current state, but this difference is inessential). For this choice of a state, Duffie et.al. (1994) prove the existence of a (stationary, conditionally spot-less) Markov equilibrium, which implies the existence of a (stationary) RE with law of motion $x_{t+1}=f\left(x_{t}, z_{t}, z_{t+1}, \sigma_{t}\right)$ and functions $c, q$, and $\theta$ defined by the corresponding projections (Duffie et.al., 1994).

\section{Two Agents and No Aggregate Risk}

We begin with two positive results on the existence question:

Proposition 1. Suppose the economy $\mathcal{E}=<\mathbf{S}, \pi, \beta, u, e, \theta_{0}, d>$ satisfies A1-A3.

i) For the debt constraint $M=0$ (no borrowing) there exists a RE with compact, finitedimensional state space (and possibly binding borrowing constraints). 
ii) If markets are effectively complete (A4 does not hold), then there is a debt constraint $M<$ $\infty$ so that a RE with finite (and therefore compact) state space and non-binding borrowing constraints exists.

Proof .

i) Follows from Duffie et.al.(1994) who prove that if short-sales of assets are prohibited ( $M=$ $0)$, a (stationary) Markov equilibrium with compact state space exists if $x_{t}=\left(\theta_{t}, c_{t}, q_{t}, z_{t}\right)$, which implies the existence of a $\mathrm{RE}$ with compact state space (see the above remark).

ii) Assume first that there exist $c_{1}, \theta_{1} \in \mathbb{R}$ with $0<c_{1}<\omega$ so that $c_{1}-e_{1}(z)=d(z) \theta_{1}$ for all $z$. Let equilibrium consumption be $c_{1}(s)=c_{1}<\omega$ and $c_{2}(s)=\omega-c_{1}$. By construction, this allocation satisfies goods market clearing. If we let asset prices be the unique bounded solution (contraction mapping theorem) to

$$
q(s)=\beta \sum_{s^{\prime}}\left(d\left(z^{\prime}\right)+q\left(s^{\prime}\right)\right) \pi\left(s^{\prime} \mid s\right),
$$

then each agent's Euler equation also holds. Suppose portfolio choices are constant $\theta_{i}(s)=\theta_{i}$. In this case, the budget constraint of agent 1 reads $\forall z: c_{1}-e_{1}(z)=d(z) \theta_{1}$. By assumption, there exists a $\theta_{1}$ so that this equation holds. If we choose $\theta_{2}=1-\theta_{1}$, the asset market clears. By Walras' law, the budget constraint of agent 1 is then satisfied. Choosing $\mathbf{S}$ as the state space, state-invariant functions for consumption and portfolio allocations, and the asset price function defined by (6), we have constructed a RE with finite (and therefore compact) state space and non-binding borrowing constraints. If there are no numbers $c_{1}, \theta$ but numbers $c_{2}, \theta_{2}$ with $0<c_{2}<\omega$ so that $c_{2}-e_{2}(z)=d(z) \theta_{2}$ for all $z$, a similar argument applies.

We now turn to the non-existence argument. The first lemma establishes that asset prices and portfolio holdings are bounded. The important point to note about lemma 1 is that the bounds are independent of the debt constraint $M$. To state the lemma, define $\left(e_{i} / d\right)_{\min } \doteq \min \left\{e_{i}(1) / d(1), \ldots, e_{i}(S) / d(S)\right\}$. 
Lemma 1. Suppose the economy $\mathcal{E}=<\mathbf{S}, \pi, \beta, u, e, \theta_{0}, d>$ satisfies A1-A3. Then for any SE (with or without binding borrowing constraints) we have:

i) There are $\underline{q}>0$ and $\bar{q}<\infty$ so that $\forall t, s^{t}: \underline{q} \leq q_{t}\left(s^{t}\right) \leq \bar{q}$. Moreover, $\underline{q}$ and $\bar{q}$ are independent of the debt constraint $M$.

ii) For all debt constraints $M$ and $\forall t, s^{t}: \theta_{i, t+1}\left(s^{t}\right) \geq-\left(e_{i} / d\right)_{\min }$.

Proof. The upper bound on the asset price is derived in Kehoe and Levine (2001). They consider an economy with a debt constraint $M=0$, but their proof immediately extends to the case with any finite borrowing constraint. Similarly, we could use the result that for the economy considered here, there are no bubbles for long-lived assets in positive net-supply (Santos and Woodford, 1997). This upper bound on asset prices depends on preferences and endowments, but not on $M$.

To construct a strictly positive lower bound, fix an equilibrium and the corresponding optimal plan of both agents. If at node $s^{t}$ we have $q_{t}\left(s^{t}\right)=\underline{q}$, then the fact that agent $i$ is choosing an optimal plan implies the following inequality:

$$
\underline{q} u_{i}^{\prime}\left(c_{i t}\left(s^{t}\right)\right) \geq \sum_{n=1}^{\infty} \beta^{n} \sum_{s^{t+n} \in \mathbf{D}\left(s^{t}\right)} u_{i}^{\prime}\left(c_{i, t+n}\left(s^{t+n}\right)\right) d\left(s_{t+n}\right) \pi\left(s^{t+n} \mid s^{t}\right) .
$$

If the inequality (7) did not hold, agent $i^{\prime} s$ plan could not be optimal because it would pay to buy one unit of the asset at node $s^{t}$ and to hold this additional unit forever consuming the dividend payments. Define $d_{\min }=\min \{d(1), \ldots, d(S)\}$. Since $c_{i, t+n}\left(s^{t+n}\right) \leq \omega$ for both agents and $c_{i t}\left(s^{t}\right) \leq \omega / 2$ for at least one agent $i$, say agent $i=1$, we must have:

$$
\underline{q} u_{1}^{\prime}(\omega / 2) \geq \frac{\beta d_{\min }}{1-\beta} u_{1}^{\prime}(\omega) .
$$

This establishes the strictly positive lower bound on the asset price.

The lower bound on portfolio holdings, $-\left(e_{i} / d\right)_{\text {min }}$, follows from the sequential budget constraint (2) and non-negativity of consumption. More specifically, if agent $i^{\prime} s$ portfolio 
holding drops below this bound, there is a history for which the corresponding sequence of portfolio holdings is unbounded from below, which violates any finite debt constraint because the asset price is bounded away from zero. To be more precise, notice first that $\theta_{i, t+1}\left(s^{t}\right)-\theta_{i t}\left(s^{t-1}\right)<-\varepsilon$ for some $\varepsilon>0$ if and only if (using the budget constraint):

$$
\frac{e_{i t}\left(z_{t}\right)-c_{i t}\left(s^{t}\right)}{q_{t}\left(s^{t}\right)}+\frac{d_{t}\left(z_{t}\right)}{q_{t}\left(s^{t}\right)} \theta_{i t}\left(s^{t-1}\right)<-\varepsilon .
$$

Since $c_{i t} \geq 0$, the inequality (9) holds if

$$
e_{i t}\left(z_{t}\right)<-d_{t}\left(z_{t}\right) \theta_{i t}\left(s^{t-1}\right)-\varepsilon q_{t}\left(s^{t}\right)
$$

Let $\hat{s}$ be the state for which $e_{i}(\hat{s}) / d(\hat{s})=\left(e_{i} / d\right)_{\min }$ and consider a history $s^{\infty}$ with $s_{\hat{t}}=$ $s_{\hat{t}+1}=\ldots=\hat{s}$. If $\theta_{i \hat{t}}\left(s^{\hat{t}-1}\right)<\left(e_{i} / d\right)_{\min }$, then at $s^{\hat{t}}$ the inequality (10) holds for small enough $\varepsilon>0$ since $q_{t}\left(s^{\hat{t}}\right) \leq \bar{q}<\infty$. Hence, $\theta_{i, \hat{t}+1}\left(s^{\hat{t}}\right)-\theta_{i \hat{t}}\left(s^{\hat{t}-1}\right)<\varepsilon$. Moreover, this and $\theta_{i \hat{t}}\left(s^{\hat{t}-1}\right)<\left(e_{i} / d\right)_{\min }$ imply that $(10)$ also holds for the subsequent node $s^{\hat{t}+1}$ for the same $\varepsilon$. More generally, (10) holds for all $s^{\hat{t}+n}, n=1,2, \ldots$ and fixed $\varepsilon$, and the portfolio sequence, $\left\{\theta_{i t}\left(s^{t}\right)\right\}$, associated with this history is therefore unbounded from below. Since the asset price is bounded away from zero, this implies that the corresponding sequence of debt, $\left\{q_{t}\left(s^{t}\right) \theta_{i, t+1}\left(s^{t}\right)\right\}$, is unbounded from below, therefore violating the debt constraint for any debt constraint $M$ at some $t=\hat{t}+T$.

The next two lemmas (lemma 2 and 3) state two properties of the equilibrium consumption set of any SE. The first lemma says that equilibrium consumption cannot be constant. The proof uses the Euler equation and budget constraint. The second lemma shows that if one agent's equilibrium consumption takes on its minimum (maximum) at one node, then it has to be equal to this minimum (maximum) for all subsequent nodes. The proof uses the Euler equation and market clearing. These two lemmas (2 and 3) imply that the equilibrium consumption set of any RE cannot be compact (lemma 4), which immediately leads to the non-existence of RE (proposition 2 and corollary 1). 
To state the next two lemmas, define for each SE the set of all possible consumption levels of agent $i: \mathbf{C}_{i} \doteq\left\{c_{i} \in \mathbb{R}_{+} \mid \exists s^{t}: c_{i t}\left(s^{t}\right)=c_{i}\right\}$. Define further the supremum $c_{i, s u p} \doteq \sup \mathbf{C}_{i}$ and infimum $c_{i, \text { inf }} \doteq \inf \mathbf{C}_{i}$, which always exist since $\mathbf{C}_{i}$ is a bounded subset of $\mathbb{R}$.

Lemma 2. Suppose the economy $\mathcal{E}=<\mathbf{S}, \pi, \beta, u, e, \theta_{0}, d>$ satisfies $A 1-A 4$. Then for any SE with non-binding borrowing constraints, individual consumption displays some fluctuations: the set $\mathbf{C}_{i}$ is not a singleton.

Proof. Suppose not, that is, there is a real number $c_{i}$ so that $c_{i t}\left(s^{t}\right)=c_{i}$ for all $s^{t}$. Notice first that solving the Euler equations forward yields the standard present value representation of asset prices (asset prices are bounded, lemma 1). For constant consumption, this implies history-independent asset prices:

$$
\begin{aligned}
\forall s^{t}: q_{t}\left(s^{t}\right) & =\sum_{n=1}^{\infty} \beta^{n} \sum_{s^{t+n} \in \mathbf{D}\left(s^{t}\right)} d\left(z_{t+n}\right) \pi\left(z_{t+n} \mid s_{t}\right) \\
& =q\left(s_{t}\right) .
\end{aligned}
$$

For any infinite history $s^{\infty}$, solving forward the sequential budget constraint (2) yields

$$
\begin{aligned}
\theta_{i t}\left(s^{t-1}\right) & =\frac{1}{q\left(s_{t}\right)+d\left(z_{t}\right)} \sum_{n=0}^{\infty}\left(\prod_{k=1}^{n} \frac{q\left(s_{t+k-1}\right)}{q\left(s_{t+k}\right)+d\left(z_{t+k}\right)}\right)\left[c_{i}-e_{i}\left(z_{t+n}\right)\right] \\
& +\left(\prod_{k=0}^{T} \frac{q\left(s_{t+k}\right)}{q\left(s_{t+k}\right)+d\left(z_{t+k}\right)}\right) \theta_{i, t+T+1}\left(s^{t+T}\right)
\end{aligned}
$$

where we adopted the convention $\prod_{k=1}^{0} x_{k}=1$. Using the fact that portfolio holdings are bounded (lemma 1) and that dividend payments are bounded away from zero, we find $\lim _{T \rightarrow \infty}\left(\prod_{k=1}^{T} \frac{q\left(s_{t+k}\right)}{q\left(s_{t+k}\right)+d\left(z_{t+k}\right)}\right) \theta_{i, t+T+1}\left(s^{t+T}\right)=0$. Hence, taking the limit $T \rightarrow \infty$ in $(12)$ leads to

$$
\theta_{i t}\left(s^{t-1}\right)=\frac{1}{q\left(s_{t}\right)+d\left(z_{t}\right)} \sum_{n=0}^{\infty}\left(\prod_{k=1}^{n} \frac{q\left(s_{t+k-1}\right)}{q\left(s_{t+k}\right)+d\left(z_{t+k}\right)}\right)\left[c_{i}-e_{i}\left(z_{t+n}\right)\right] .
$$

The series (13) converges since

$$
\lim _{T \rightarrow \infty}\left(\prod_{k=1}^{n} \frac{q\left(s_{t+k-1}\right)}{q\left(s_{t+k}\right)+d\left(z_{t+k}\right)}\right)\left[c_{i}-e_{i}\left(z_{t+n}\right)\right]
$$




$$
\begin{aligned}
& =\lim _{T \rightarrow \infty} \frac{q\left(s_{t}\right)}{q\left(s_{t+n}\right)}\left(\prod_{k=1}^{n} \frac{q\left(s_{t+k}\right)}{q\left(s_{t+k}\right)+d\left(z_{t+k}\right)}\right)\left[c_{i}-e_{i}\left(z_{t+n}\right)\right] \\
& =0
\end{aligned}
$$

Notice that (14) holds because asset payoffs and prices are bounded away from zero and bounded from above. Since the right-hand-side of (13) neither depends on $s^{t-1}$ nor explicitly on $t$, equation (13) says that portfolio choices are time- and state-independent: $\theta_{i t}\left(s^{t-1}\right)=\bar{\theta}_{i}$ for all $s^{t-1}$. In this case the sequential budget constraint reads

$$
\forall z_{t}: c_{i}-e_{i}\left(z_{t}\right)=d\left(z_{t}\right) \bar{\theta}_{i}
$$

which does not hold because of A4.

Remark 2. Lemma 2 also shows that for the incomplete-markets economy analyzed in this paper, the equilibrium is never efficient. Kuebler and Schmedders (2001) analyze a framework more general than the model considered in this paper, and show that for a generic set of economies (which might not contain the constant-aggregate-endowment case) the equilibrium is Pareto inefficient.

The next lemma contains the paper's main idea: if consumption attains one of the extreme points of the equilibrium consumption set, then it has to stay there forever. The argument heavily relies on the fact that first-order conditions (Euler equations) have to hold with equality when consumption is strictly positive and borrowing constraints are not binding.

Lemma 3. Suppose the economy $\mathcal{E}=<\mathbf{S}, \pi, \beta, u, e, \theta_{0}, d>$ satisfies $A 1$-A3. Then for any SE with non-binding borrowing constraints, minimal (maximal) consumption for agent $i$ at a particular node implies minimal (maximal) consumption at all subsequent nodes: for any SE, if there exists a node $s^{t}$ so that $c_{i t}\left(s^{t}\right)=c_{i, \text { inf }}$, then $c_{i, t+n}\left(s^{t+n}\right)=c_{i, \text { inf }}$ for all subsequent nodes $s^{t+n} \in \mathbf{D}\left(s^{t}\right)$; for any $S E$, if there exists a node $s^{t}$ so that $c_{i t}\left(s^{t}\right)=c_{i, \text { sup }}$, then $c_{i, t+n}\left(s^{t+n}\right)=c_{i, \text { sup }}$ for all subsequent nodes $s^{t+n} \in \mathbf{D}\left(s^{t}\right)$. 
Proof . Fix a SE. If borrowing constraints do not bind, the Euler equations are given by (4). Define a new measure through

$$
\tilde{\pi}\left(s_{t+1} \mid s^{t}\right) \doteq \frac{\left[q_{t+1}\left(s^{t+1}\right)+d\left(z_{t+1}\right)\right] \pi\left(s_{t+1} \mid s_{t}\right)}{\sum_{s_{t+1}}\left[q_{t+1}\left(s^{t+1}\right)+d\left(z_{t+1}\right)\right] \pi\left(s_{t+1} \mid s_{t}\right)} .
$$

For any $s^{t}$, the two measures $\pi\left(. \mid s_{t}\right)$ and $\tilde{\pi}\left(. \mid s^{t}\right)$ are equivalent measures because asset prices and dividends are strictly positive. With this definition, the Euler equations (4) become

$$
\forall i: \quad q_{t}\left(s^{t}\right) u_{i}^{\prime}\left(c_{i t}\left(s^{t}\right)\right)=\beta \sum_{s_{t+1}} u_{i}^{\prime}\left(c_{i, t+1}\left(s^{t+1}\right)\right) \tilde{\pi}\left(s_{t+1} \mid s^{t}\right) .
$$

Clearly, (16) are the Euler equations for a one-period, risk-free bond.

Suppose now that there is a $s^{t}$ with $c_{1 t}\left(s^{t}\right)=c_{1, i n f}$. Fix this node $s^{t}$. Because of market clearing and the assumption of no aggregate risk, we also have $c_{2 t}\left(s^{t}\right)=c_{2, \text { sup }}$. Since marginal utility functions are non-increasing in consumption, the Euler equations (16) at $s^{t}$ imply

$$
\begin{aligned}
\text { i) } q_{t}\left(s^{t}\right) & \leq \beta \\
\text { ii) } q_{t}\left(s^{t}\right) & \geq \beta .
\end{aligned}
$$

In words: the fact that agent 1 expects an increase in consumption (he has hit bottom) imposes an upper bound on the asset price (a lower bound on the interest rate) and the fact that agent 2 expects a decrease in consumption imposes a lower bound on the asset price (an upper bound on the interest rate).

Clearly, (17) only holds if $q_{t}\left(s^{t}\right)=\beta$. But then the Euler equations (16) imply $c_{1, t+1}\left(s^{t+1}\right)=$ $c_{1, \text { inf }}$ for all $s^{t+1} \in \mathbf{D}\left(s^{t}\right)$ and $c_{2, t+1}\left(s^{t+1}\right)=c_{2, \text { sup }}$ for all $s^{t+1} \in \mathbf{D}\left(s^{t}\right)$. This must hold for all $s^{t+1} \in \mathbf{D}\left(s^{t}\right)$ because of assumption A1 (all transition probabilities are strictly positive) and the fact that $\pi$ and $\tilde{\pi}$ are equivalent measures. An identical argument shows that $c_{1, t+2}\left(s^{t+2}\right)=c_{1, \text { inf }}$ for all $s^{t+2} \in \mathbf{D}\left(s^{t}\right)$ and $c_{2, t+2}\left(s^{t+2}\right)=c_{2, \text { sup }}$ for all $s^{t+2} \in \mathbf{D}\left(s^{t}\right)$. More generally, $c_{1 t}\left(s^{t}\right)=c_{1, \text { inf }}$ implies $c_{1, t+n}\left(s^{t+n}\right)=c_{1, \text { inf }}$ for all $s^{t+n} \in \mathbf{D}\left(s^{t}\right)$ and $c_{2 t}\left(s^{t}\right)=c_{2, \text { sup }}$ implies $c_{2, t+n}\left(s^{t+n}\right)=c_{2, \text { sup }}$. An analogous argument shows that $c_{1 t}\left(s^{t}\right)=c_{1, \text { sup }}$ implies 
$c_{1, t+n}\left(s^{t+n}\right)=c_{1, \text { sup }}$ for all $s^{t+n} \in \mathbf{D}\left(s^{t}\right)$ and $c_{2 t}\left(s^{t}\right)=c_{2, \text { inf }}$ implies $c_{2, t+n}\left(s^{t+n}\right)=c_{2, \text { inf }}$ for all $s^{t+n} \in \mathbf{D}\left(s^{t}\right)$.

The final lemma combines lemma 2 and 3 to derive that a statement about the nature of RE. In order to state the lemma, define for a particular RE the equilibrium consumption set of agent $i$ as $\tilde{\mathbf{C}}_{i} \doteq\left\{c_{i} \in \mathbb{R}_{+} \mid \exists(x, s) \in \mathbf{X} \times \mathbf{S}: c_{i}(x, s)=c_{i}\right\}$. Clearly, the equilibrium consumption set of a $\mathrm{RE}$ is the union of the equilibrium consumption sets of those $\mathrm{SE}$ associated with the RE.

Lemma 4. Suppose the economy $\mathcal{E}=<\mathbf{S}, \pi, \beta, u, e, \theta_{0}, d>$ satisfies assumptions A1-A4. Then for any RE with non-binding borrowing constraints, the equilibrium consumption set, $\tilde{\mathbf{C}}_{i}$, is not compact (closed).

Proof. Suppose not, that is, suppose $\tilde{\mathbf{C}}_{i}$ is compact. Then $\tilde{\mathbf{C}}_{i}$ contains its supremum: $\tilde{c}_{i, \text { sup }} \in \tilde{\mathbf{C}}_{i}$. Put differently, there exists a state $(\hat{x}, \hat{s}) \in \mathbf{X} \times \mathbf{S}$ with $c_{i}(\hat{x}, \hat{s})=\tilde{c}_{i, \text { sup }}$. Each $\mathrm{RE}$ is associated with a family of $\mathrm{SE}$, one for each initial state, $\left(x_{0}, s_{0}\right) \in \mathbf{X} \times \mathbf{S}$. For the $\mathrm{SE}$ with initial state $\left(x_{0}, s_{0}\right)=(\hat{x}, \hat{s})$, we have that $c_{i, \text { sup }}=\tilde{c}_{i, \text { sup }}$, which implies that in this SE consumption of agent $i$ in period $t=0$ must be maximal: $c_{i 0}=c_{i}\left(x_{0}, s_{0}\right)=\tilde{c}_{i, \text { sup }}=c_{i, \text { sup }}$. Lemma 3 then implies that for this particular SE, consumption of agent $i$ is maximal in all subsequent periods: $c_{i t}\left(s^{t}\right)=c_{i, \text { sup }} \forall s^{t}$. But this contradicts lemma 2 .

Proposition 2. Suppose the economy $\mathcal{E}=<\mathbf{S}, \pi, \beta, u, e, \theta_{0}, d>$ satisfies $A 1-A 4$. Then there is no RE with non-binding borrowing constraints that is defined by continuous functions, $f, q, c, \theta$, on a compact domain, $\mathbf{X} \times \mathbf{S}$.

Proof. The equilibrium consumption set, $\tilde{\mathbf{C}}_{i}$, is the image set of the consumption policy function, $c_{i}: \mathbf{X} \times \mathbf{S} \rightarrow \mathbb{R}_{+}$. If this function is continuous with compact domain, its image set is compact. This contradicts lemma 4.

Using lemma 1, we can rephrase proposition 2 as follows: 
Corollary 1. Suppose the economy $\mathcal{E}=<\mathbf{S}, \pi, \beta, u, e, \theta_{0}, d>$ satisfies A1-A4. Then there is no RE with debt constraint $M>\bar{q} \max \left\{\left(e_{1} / d\right)_{\min },\left(e_{2} / d\right)_{\min }\right\}$ that is defined by continuous functions, $f, q, c, \theta$, on a compact domain, $\mathbf{X} \times \mathbf{S}$.

Proof. Lemma 1 says that for arbitrary, but finite, debt constraint, $M$, agent $i$ will only choose a portfolio plan that satisfies $q_{t}\left(s^{t}\right) \theta_{i, t+1}\left(s^{t}\right) \geq-q_{t}\left(s^{t}\right)\left(e_{i} / d\right)_{\min }$ for all $s^{t}$. Thus, we have $q_{t}\left(s^{t}\right) \theta_{i, t+1}\left(s^{t}\right) \geq-\bar{q}\left(e_{i} / d\right)_{\min }$ for all $s^{t}$. This means that for any $M>\bar{q}\left(e_{i} / d\right)_{\min }$, agent $i^{\prime} s$ borrowing constraint never binds. But if the borrowing constraint never binds, then proposition 2 says that no RE exists.

Remark 3. If $\beta_{1} \neq \beta_{2}$, then the proof of non-existence follows similar lines. The proofs of lemma 2 and 3 go through without change, and lemma 4 is replaced by the statement that either $c_{i, \text { sup }} \notin \mathbf{C}_{i}$ or $c_{i, \text { inf }} \notin \mathbf{C}_{i}$.

\section{Extensions}

In this section, we discuss possible extensions of the non-existence argument. We begin with the extension to more than two agents. The definition of SE and RE provided in section 2 immediately extends to the case of any finite number, $I$, of agents. Although the non-existence result stated below also applies to economies with a continuum of agents, to save space the argument is presented for the case of a finite number of agents. Clearly, with the exception of lemma 3 , all results derived in the last section still hold for more than two agents. The analog of lemma 3 for the case of more than two agents is:

Lemma 5. Suppose the economy $\mathcal{E}=<\mathbf{S}, \pi, \beta, u, e, \theta_{0}, d>$ satisfies $A 1$ - $A 3$ and there are at least two agents, $I \geq 2$. If the marginal utility function, $u_{i}^{\prime}$, of each agent $i=1, \ldots, I$ is convex, then in any SE with non-binding borrowing constraints, minimal consumption for one agent $i$ at a particular node implies minimal consumption for this agent at all subsequent 
nodes: for any $S E$, if there exists a node $s^{t}$ so that $c_{i t}\left(s^{t}\right)=c_{i, \text { inf }}$, then $c_{i, t+n}\left(s^{t+n}\right)=c_{i, \text { inf }}$ for all subsequent nodes $s^{t+n} \in \mathbf{D}\left(s^{t}\right)$.

Proof. Without loss of generality, we choose $i=1$. Fix a SE with non-binding borrowing constraints and suppose there exists a node $s^{t}$ for which $c_{1 t}\left(s^{t}\right)=c_{1, \text { inf }}$. We fix this node $s^{t}$ throughout the analysis. After introducing an equivalent measure, the Euler equations at $s^{t}$ are given by (16). The Euler equation for agent $i=1$ again implies

$$
q_{t}\left(s^{t}\right) \leq \beta
$$

Since $c_{1 t}\left(s^{t}\right)=c_{1, \text { inf }}$ and there is no aggregate risk, there exists a $\hat{i} \in\{2, \ldots, I\}$ with

$$
c_{\hat{i} t}\left(s^{t}\right) \geq \sum_{s_{t+1}} c_{\hat{i}, t+1}\left(s^{t+1}\right) \tilde{\pi}\left(s_{t+1} \mid s^{t}\right) .
$$

The proof of (19) is shown below. Since the marginal utility function is non-increasing and convex, we have (using 19):

$$
\begin{aligned}
q_{t}\left(s^{t}\right) & =\beta \frac{\sum_{s_{t+1}} u_{\hat{i}}^{\prime}\left(c_{\hat{i}, t+1}\left(s^{t+1}\right)\right) \tilde{\pi}\left(s_{t+1} \mid s^{t}\right)}{u_{\hat{i}}^{\prime}\left(c_{\hat{i} t}\left(s^{t}\right)\right.} \\
& \geq \beta \frac{u_{\hat{i}}^{\prime}\left(\sum_{s_{t+1}} c_{\hat{i}, t+1}\left(s^{t+1}\right) \tilde{\pi}\left(s_{t+1} \mid s^{t}\right)\right)}{u_{\hat{i}}^{\prime}\left(c_{\hat{i} t}\left(s^{t}\right)\right.} \\
& \geq \beta .
\end{aligned}
$$

Clearly, (18) and (20) can only hold if $q_{t}\left(s^{t}\right)=\beta$, which implies $c_{1, t+1}\left(s^{t+1}\right)=c_{1, \text { inf }}$ for all $s^{t+1} \in \mathbf{D}\left(s^{t}\right)$. An identical argument shows that $c_{1, t+2}\left(s^{t+2}\right)=c_{1, \text { inf }}$ for all $s^{t+2} \in \mathbf{D}\left(s^{t}\right)$. More generally, $c_{1 t}\left(s^{t}\right)=c_{1, \text { inf }}$ implies $c_{1, t+n}\left(s^{t+n}\right)=c_{1, \text { inf }}$ for all $s^{t+n} \in \mathbf{D}\left(s^{t}\right)$.

It remains to be shown that (19) holds. Notice first that market clearing reads

$$
\begin{aligned}
\sum_{i=2}^{I} c_{i t}\left(s^{t}\right) & =\omega-c_{1, \text { inf }} \\
\forall s^{t+1} \in \mathbf{D}\left(s^{t}\right): \sum_{i=2}^{I} c_{i, t+1}\left(s^{t+1}\right) & =\omega-c_{1, t+1}\left(s^{t+1}\right) .
\end{aligned}
$$


Equation (19) implies

$$
\forall s^{t+1} \in \mathbf{D}\left(s^{t}\right): \sum_{i=2}^{I} c_{i t}\left(s^{t}\right)-\sum_{i=2}^{I} c_{i, t+1}\left(s^{t+1}\right)=c_{1, t+1}\left(s^{t+1}\right)-c_{1, i n f},
$$

which in turn implies

$$
\sum_{i=2}^{I} c_{i t}\left(s^{t}\right) \geq \sum_{i=2}^{I} \sum_{s_{t+1}} c_{i, t+1}\left(s^{t+1}\right) \tilde{\pi}\left(s_{t+1} \mid s^{t}\right) .
$$

Clearly, (23) can only hold if (19) holds.

Proposition 2 and the corollary 1 now read as follows:

Proposition 3. Suppose the economy $\mathcal{E}=<\mathbf{S}, \pi, \beta, u, e, \theta_{0}, d>$ satisfies $A 1$ - $A 4$ and there are at least two agents, $I \geq 2$. If agents' marginal utility functions are all convex, then there is no RE with non-binding borrowing constraints that is defined by continuous functions, $f, q, c, \theta$, on a compact domain, $\mathbf{X} \times \mathbf{S}$.

Corollary 2. Suppose the economy $\mathcal{E}=<\mathbf{S}, \pi, \beta, u, e, \theta_{0}, d>$ satisfies A1-A4 and there are at least two agents, $I \geq 2$. If agents' marginal utility functions are all convex, then there is no RE with debt constraint $M>\bar{q} \max \left\{\left(e_{1} / d\right)_{\min }, \ldots,\left(e_{I} / d\right)_{\min }\right\}$ that is defined by continuous functions, $f, q, c, \theta$, on a compact domain, $\mathbf{X} \times \mathbf{S}$.

Next we discuss an assumptions on preferences that leads to non-existence for all endowment vectors (including those with a large amount of aggregate risk). Interestingly, this is the preference assumption most often made in macroeconomics.

Assumption 5. Agents have identical CRRA-preferences with degree of relative risk aversion greater or equal to one: $u_{i}\left(c_{i t}\right)=c_{i t}^{1-\gamma} /(1-\gamma), \gamma>1$, or $u_{i}\left(c_{i t}\right)=\log c_{i t}$.

Proposition 4. Suppose the economy $\mathcal{E}=<\mathbf{S}, \pi, \beta, u, e, \theta_{0}, d>$ satisfies assumptions A1,A2,A4, and A5 (but not necessarily assumption A3) and there are at least two agents, $I \geq 2$. Then there is no $R E$ with non-binding borrowing constraints that is defined by 
continuous functions, $f, q, c, \theta$, on a compact domain, $\mathbf{X} \times \mathbf{S}$.

Proof. With this preference specification, the equilibrium conditions (5) for an economy with aggregate risk can be transformed into the equilibrium conditions of an economy with

constant (unit) aggregate endowment and effective discount factor $\tilde{\beta}=\beta \omega_{t}^{-\gamma}$. Simply introduce the scaled variables $\tilde{c}_{i t}=c_{i t} / \omega_{t}, \tilde{e}_{i t}=e_{i t} / \omega_{t}, \tilde{d}_{t}=d_{t} / \omega_{t}$, and $\tilde{q}_{t}=q_{t} / \omega_{t}$. This new economy is an economy with no aggregate risk and common, multiplicative taste shocks, which has no recursive equilibrium with non-binding borrowing constraints for the following reason. Step 1 (lemma 3) goes through because constant consumption still implies constant portfolio holdings (even though it does not imply constant prices), which in turn violates the budget constraint. Step 2 (lemma 4) remains almost unchanged because common taste shocks disappear from the equilibrium conditions once an appropriate equivalent measure is introduced. Because for this preference specification the marginal utility function is strictly convex, proposition 3 applies and the non-existence holds for any number of agents.

Corollary 3. Suppose the economy $\mathcal{E}=<\mathbf{S}, \pi, \beta, u, e, \theta_{0}, d>$ satisfies assumptions A1,A2,A4, and A5 (but not necessarily assumption A3) and there are at least two agents, $I \geq 2$. Then there is no $R E$ with debt constraint $M>\bar{q} \max \left\{\left(e_{1} / d\right)_{\min }, \ldots,\left(e_{I} / d\right)_{\min }\right\}$ that is defined by continuous functions, $f, q, c, \theta$, on a compact domain, $\mathbf{X} \times \mathbf{S}$.

\section{Concluding Remarks}

This paper showed that for incomplete-market economies with no aggregate risk and utility functions that are unbounded from below, no RE (ME) with compact state space and non-binding borrowing constraints exists. This conclusion discusses possible extensions of the non-existence result in addition to the extensions discussed in the previous Section.

A straightforward (but lengthy) extension of the argument put forward in this paper 
shows that the same type of non-existence result holds for an open set of economies with small amount of aggregate risk. In other words, non-existence is robust with respect to perturbations of endowments. For this type of extension, lemma 2 has to be sharpened to the statement that there is a lower bound on consumption fluctuations that is bounded away from zero, and the new version of lemma 3 would state that future consumption fluctuations can be made arbitrarily small. An essential requirement for this argument to be valid is that all functions involved are continuous functions restricted to a compact set, and are therefore bounded. A similar extension is possible for small taste shocks.

The non-existence argument does not extend, however, to a non-compact state space because this could lead to marginal utility functions that are unbounded even if restricted to the equilibrium domain. Thus, this paper cannot rule out that there are RE (ME) with non-compact state space and non-binding borrowing constraints for which the implied equilibrium consumption set is $\tilde{\mathbf{C}} \equiv\left\{\left(c_{1}, c_{2}\right) \in \mathbb{R}_{++}^{2} \mid c_{1}+c_{2}=\omega\right\}$.

In the case of bounded utility functions the contradiction leading to the non-existence result might be avoided by including zero consumption in the equilibrium consumption set as an isolated point. As mentioned in footnote 7, zero consumption could be an equilibrium outcome even if an Inada condition is satisfied (but not with utility unbounded from below) when an agent begins his life with so much debt that his net-endowment is zero: $\theta_{i 0}+e_{i}\left(z_{0}\right)=$ 0. In this case the agent's choice set at the initial node is a singleton and no Euler equation has to hold for this agent at the initial node (any further increase in debt will violate the budget constraint and the agent is forced to consume $\left.c_{i 0}=0\right)$. Clearly, such an approach to the existence of $\mathrm{RE}(\mathrm{ME})$ requires one to abandon the common assumption (Hernandez and Santos, 1996, Levine and Zame, 1996, Magill and Quinzii, 1994,96) that net-endowments are strictly positive at all date-events. ${ }^{8}$

\footnotetext{
${ }^{8}$ To the best of my knowledge, RE (ME) of this type have not been studied by the literature.
} 


\section{References}

Aiyagari, R. (1994) "Uninsured Idiosyncratic Risk and Aggregate Saving," Quarterly Journal of Economics 109, 659-84.

Atkeson, A. and R. Lucas (1992) "On the Efficient Distribution with Private Information," Review of Economic Studies 59, 427-453.

Becker, R. and I. Zilcha (1997) "Stationary Ramsey Equilibria Under Uncertainty," Journal of Economic Theory 75, 122-141.

Cooley, T. and E. Prescott (1995), "Economic Growth and Business Cycles," in Frontiers of Business Cycle Research, T. Cooley, ed., Princeton University Press, Princeton, New Jersey.

Duffie, D., J. Geanakoplos, A. Mas-Colell, and A. McLennan (1994) "Stationary Markov Equilibria," Econometrica 62, 745-781.

Hart, O. (1975) "On the Optimality of Equilibrium When the Market Structure is Incomplete," Journal of Economic Theory 9, 293-311.

Heaton, J. and D. Lucas (1996) "Evaluating the Effects of Incomplete Markets on Risk Sharing and Asset Pricing," Journal of Political Economy 104, 443-487.

Hernandez, A., and M. Santos (1996) "Competitive Equilibria for Infinite-Horizon Economies with Incomplete Markets," Journal of Economic Theory 71, 102-130.

Huggett, M. (1993) "The Risk-free Rate in Heterogeneous-Agent Incomplete-Market Economies," Journal of Economic Dynamics and Control 17, 953-969.

Kehoe, T. and D. Levine (2001) "Liquidity Constraint Markets versus Debt Constrained Markets," Econometrica 69: 575-598.

Krusell, P. and A. Smith (1998) Income and Wealth Heterogeneity in the Macroeconomy," Journal of Political Economy 106, 867-896.

Kuebler, F. and K. Schmedders (2001), "Generic Inefficiency of Equilibria in the Generalized Lucas Asset Pricing Model,"Economic Theory, forthcoming.

Kuebler, F. and K. Schmedders (2002), "Recursive Equilibria in Economies with Incom- 
plete Markets, "Macroeconomic Dynamics 6.

Levine, D., and W. Zame (1996) "Debt Constraints and Equilibrium in Infinite-Horizon Economies with Incomplete Markets," Journal of Mathematical Economics 26, 103-131.

Ljungqvist, L. and T. Sargent Recursive Macroeconomic Theory, MIT Press, Cambridge, Mass., 2000.

Lucas, R. (1978) "Asset Prices in an Exchange Economy," Econometrica 46, 1429-45.

Magill, M., and M. Quinzii (1994) "Infinite Horizon Incomplete Markets, "Econometrica $62,853-880$.

Magill, M. and M. Quinzii (1996) "Incomplete Markets Over an Infinite Horizon"LongLived Securities and Speculative Bubbles, "Journal of Mathematical Economics 26, 133-170.

Radner, R. (1972) "Existence of Equilibrium of Plans, Prices, and Price Expectations in a Sequence of Markets, "Econometrica 40, 289-303

Santos, M. (2000) "On Non-Existence of Markov Equilibria in Competitive-Market Economics," Journal of Economic Theory, forthcoming.

Santos, M. and M. Woodford (1997) "Rational Asset Pricing Bubbles," Econometrica 65, $19-59$.

Spear, S. (1985) "Rational Expectations in the Overlapping Generations Model," Journal of Economic Theory 35, 251-275.

Stokey, N. and R. Lucas, with E. Prescott,Recursive Methods in Economic Dynamics, Harvard University Press, 1989.

Thomas, J. and T. Worrall (1990) "Income Fluctuations and Asymmetric Information: An Example of a Repeated Principal-Agent Problem", Journal of Economic Theory 51, 367-390. 